\title{
An unexpected case report of Ascaris lumbricoides in pregnant woman
}

\author{
Valentino Granero, ${ }^{1,2}$ Daniela Dompè, ${ }^{3}$ Elvio Peyronel, ${ }^{2}$ Cristina Crocillà, ${ }^{2}$ Maria Rita Cavallo ${ }^{2}$ \\ ${ }^{1}$ Immunohaematology and Transfusion Medicine, Local Health Unit, Asti; ${ }^{2}$ Unified Analysis Laboratory Rivoli-Pinerolo, \\ Local Health Unit TO3, Pinerolo; ${ }^{3}$ Obstetrics and Gynaecology, Local Health Unit TO3, Pinerolo, Italy
}

\begin{abstract}
Summary
Soil-transmitted helminths, primarily Ascaris, Trichuris and hookworm, infect more than 760 million people worldwide. Parasitic diseases represent a social and economic problem in developing countries. Herein, we present a case of ascariasis in a pregnant woman, who was treated with mebendazole for three days without teratogenic effects. It is emphasized the need not to underestimate helminth infections in developed countries and how rapid notification is needed in order to quickly establish a guided therapy especially in pregnant women.
\end{abstract}

\section{Introduction}

Soil-trasmitted helminths (SHTs) include Ascaris lumbricoides, Trichuris trichiura and hookworm (Necator americanus and/or Ancylostoma duodenale) (7). Human ascariasis infects over

Correspondence: Valentino Granero, S.C. Immunoematologia e Medicina Trasfusionale ASL AT, C.so Dante Alighieri 202, Asti (AT), Italy.

Tel.: +39.0141.485401 - Fax: +39.0141 .485422 .

E-mail:vgranero@asl.at.it

Key words: Helminth infection, pregnancy, Anthelminthic.

Contributions: VG: first author, microbiologist during the case report; DD: author, physician during the case report; EP: author, microbiologist during the case report; $\mathrm{CC}$ : author, microbiologist during the case report; MRC: author, director of laboratory analysis during the case report.

Conflict of interest: the authors declare no potential conflict of interest.

Funding: none.

Received for publication: 30 May 2018.

Revision received: 13 February 2019.

Accepted for publication: 14 February 2019.

(C) Copyright V. Granero et al., 2019

Licensee PAGEPress, Italy

Microbiologia Medica 2019; 34:7585

doi:10.4081/mm.2019.7585

This article is distributed under the terms of the Creative Commons Attribution Noncommercial License (by-nc 4.0) which permits any noncommercial use, distribution, and reproduction in any medium, provided the original author(s) and source are credited.
760 million people worldwide, mostly children, and the disease ranging from mild to severe and even fatal cases (9).

Parasites cause high morbidity by leading to malnutrition, irondeficiency anaemia, malabsorption, intestinal obstruction, and mental and physical growth retardation in childhood. Parasitic diseases represent a social and economic problem in developing countries (8).

STH infections are commonly treated with the benzimidazole drugs albendazole or mebendazole, which are considered safe after the first trimester of pregnancy (3). These drugs are contraindicated during the first trimester of pregnancy due to potential teratogenicity; however, there is little evidence of teratogenic effects in humans resulting from taking appropriate doses of these drugs in early pregnancy (10).

We report a case of Ascaris lumbricoides in a six weeks' pregnant woman who has been treated with mebendazole after infectivologist consultancy.

\section{Case Report}

The patient was a resident in Pinerolo's territory of 32-yearold pregnant woman (early pregnancy) with an important history of two abortions in the last year. She is a kindergarten teacher without relevant previous infections but she been suffering of hypothyroidism and is treated with $50 \mu \mathrm{g}$ of sodium levothyroxine. At admission to our emergency department, the patient presented good general condition without fever, without abdominal pain, (heart rate $75 \mathrm{bpm}$, temperature $36.8^{\circ} \mathrm{C}$ ) and she reported no complaints of any allergies. At the visit the pregnancy is in evolution, ultrasound measurements of crown rump length (CRL) and gestational sac diameter are regular. The patient said that in the lasting three days she had diarrhea, no symptom of urinary infection. The patient explained us that in the night she found a parasite in feces. Our microbiologist identified an Ascaris lumbricoides. No other blood tests were performed.

Subsequently, after infectivologist consultancy, she was treated with mebendazole (100 mg for 3 days) without side effects and negative outcomes on the conception product.

The following week, the patient was afebrile, without diarrhea, with absence of uterine contractions and vaginally bleeding, ultrasound measurements were regular.

\section{Discussion and Conclusions}

STH infections are common worldwide and contribute to a high burden of malnutrition and morbidity in poor tropical and subtropical regions, where environmental control measures such as adequate sanitation are limited (9). 
Transmission of geo-helminths is most efficient in areas with poor sanitation, where the risk of faecal contamination of soil is high (5). The three common STHs share a similar lifecycle, whereby worms are ingested and subsequently inhabit the intestine. In the gut they reproduce and deposit eggs, which are then shed in faeces and introduced into the environment. The STH's life cycle continues in the human host through either ingestion of larvae in the case of Ascaris and Trichuris, or penetration of the skin by the larvae in the case of hookworms.

The morbidity from these infections and the rate of transmission are directly related to the number of worms harboured in the host, intensity of infection is the main epidemiological index used to describe soil-transmitted helminth infection (1). Intensity of

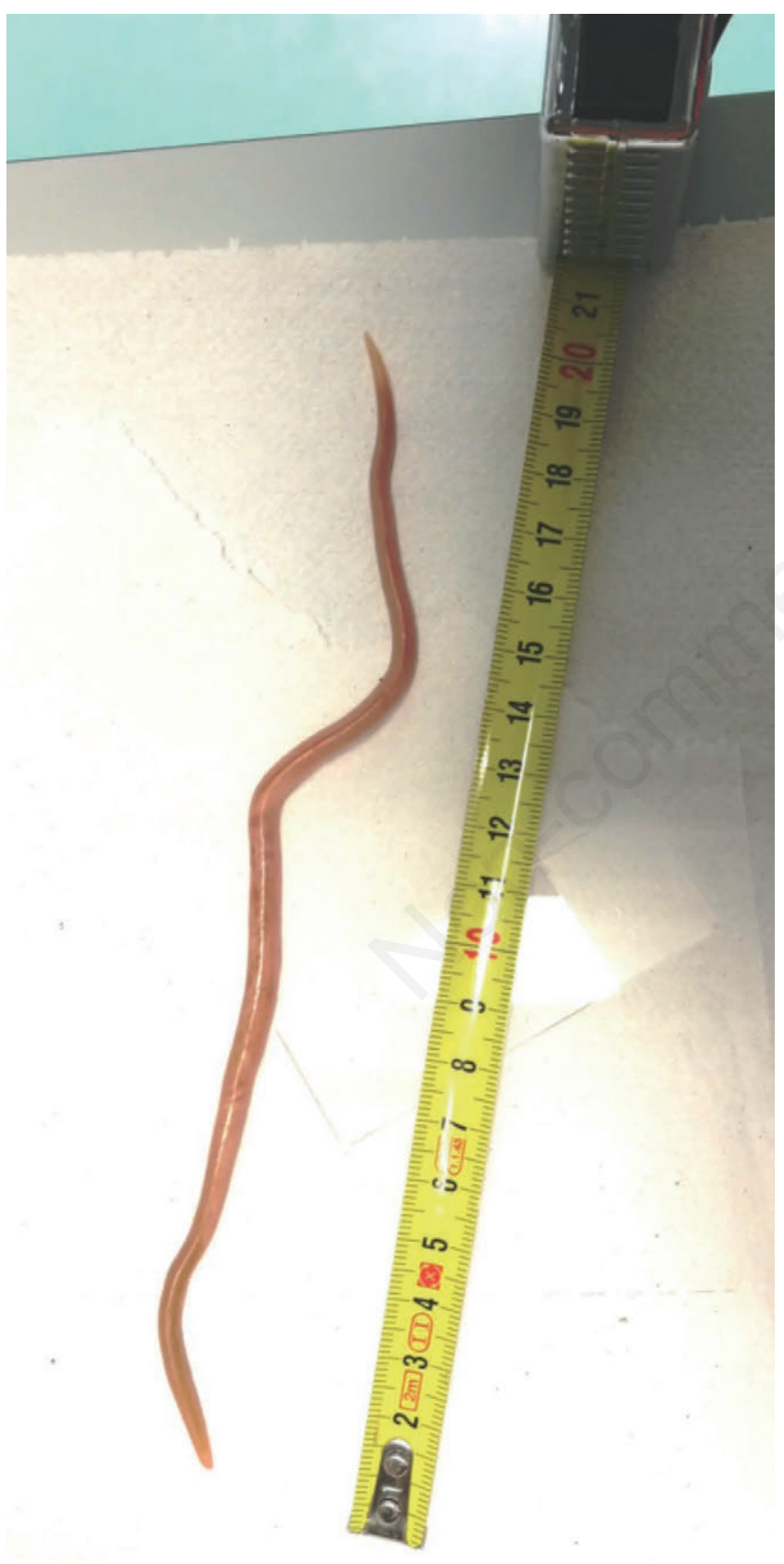

Figure 1. Ascaris lumbricoides larva. infection is measured by the number of eggs per gram of faeces, generally by the Kato-Katz faecal thick-smear technique (6).

In Italy, although the era of great migrations, the SHT have not disappeared and the prevalence is less than 5\% (4).

The diagnostic approach is based on the specific epidemiological and clinical indications and the minimum investigation that each laboratory must guarantee is the standard copro-parasitological examination through the vision of the preparation (2).

In this case, there was microbiological evidence of the presence of larvae of Ascaris lumbricoides excreted in the faeces (Figure 1), however without having had previous opportunities for diagnosis through parasitological examination. Moreover, it was not possible to verify the eventual eosinophilia with hemochrome (1).

Despite the first trimester of pregnancy, this report shows that the risk of developing SHT infections is global and can also affect pregnant women in developed countries (10). In addition, therapeutic treatment with mebendazole should only be administered after medical prescription at an appropriate dose to avoid teratogenic effects on the embryo $(3,10)$.

In conclusion, this is an autochthonous case linked to the work activity of the patient who through probable poor hand hygiene has contracted the infection. It suggests how important early diagnosis is to guide the best therapeutic approach even if SHTs can be widely recognized (2).

\section{References}

1. Bethony J, Brooker S, Albonico S, et al. Soil-transmitted helminth infections: ascariasis, trichuriasis, and hookworm. Lancet 2006;367:1521-32.

2. Bracaglia G, Mancinelli L, Ranno S, et al. Globalization effects on the reports of non-endemic parasitosis in Italy. Microbiol Med 2018;33:7932.

3. Halder JB, Benton J, Julé AM, et al. Systematic review of studies generating individual participant data on the efficacy of drugs for treating soil-transmitted helminthiases and the case for data-sharing. PLOS Negl Trop Dis 2017;11:e0006053.

4. Grande R, Ranzi ML, Restelli A, et al. Prevalenza delle parassitosi intestinali nella popolazione afferente alla Fondazione IRCC Cà Granda, Ospedale Maggiore Policlinico di Milano: confronto tra il 1984-1985 e il triennio 2007-2009. Infez Med 2011;1:28-38.

5. Imhoff-Kunsch B, Briggs V. Antihelminthics in Pregnancy and Maternal, Newborn and Child Health. Paediatr Perinat Epidemiol 2012;26:223-38.

6. Katz N, Chaves A, Pellegrino J. A simple device for quantitative stool thick-smear technique in schistosomiasis mansoni. Rev Inst Med Trop Sao Paulo 1972;14:397-400.

7. Lamberton PHL, Jourdan PM. Human Ascarias: Diagnostics Update. Curr Trop Med Rep 2015;2:189-200.

8. Manganelli L, Berrilli F, Di Cave D, et al. Intestinal parasite infections in immigrant children in the city of Rome, related risk factors and possible impact on nutritional status. Paras Vectors 2012;5:265.

9. Pullan RL, Smith JL, Jasrasaria R, et al. Global numbers of infection and disease burden of soil transmitted helminth infections in 2010. Parasi Vectors 2014;7:37.

10. Torp-Pedersen A, Jimenez-Solem E, Cejvanovic V, et al. Birth outcomes after exposure to mebendazole and pyrvinium during pregnancy - A Danish nationwide cohort study. J Obstet Gynaecol 2016;36:1020-5. 